\title{
Random, Messy, Funny, Raw: Finstas as Intimate Reconfigurations of Social Media
}

\author{
Sijia Xiao' ${ }^{1}$, Danaë Metaxa², Joon Sung Park ${ }^{3}$, \\ Karrie Karahalios ${ }^{3}$, Niloufar Salehi ${ }^{1}$ \\ ${ }^{1}$ University of California, Berkeley, ${ }^{2}$ Stanford University, ${ }^{3}$ University of Illinois at Urbana-Champaign
\{xiaosijia, nsalehi $\} @$ berkeley.edu, metaxa@ @ stanford.edu, \{jp19, kkarahal $\} @$ illinois.edu
}

\begin{abstract}
Among many young people, the creation of a finsta - a portmanteau of "fake" and "Instagram" which describes secondary Instagram accounts-provides an outlet to share emotional, low-quality, or indecorous content with their close friends. To study why people create and maintain finstas, we conducted a qualitative study through interviews with finsta users and content analysis of video bloggers exposing their finsta on YouTube. We found that one way that young people deal with mounting social pressures is by reconfiguring online platforms and changing their purposes, norms, expectations, and currencies. Carving out smaller spaces accessible only to close friends allows users the opportunity for a more unguarded, vulnerable, and unserious performance. Drawing on feminist theory, we term this process intimate reconfiguration. Through this reconfiguration, finsta users repurpose an existing and widely-used social platform to create opportunities for more meaningful and reciprocal forms of social support.
\end{abstract}

\section{Author Keywords}

Performance; reconfiguration; feminist HCI; finsta

\section{CCS Concepts}

-Human-centered computing $\rightarrow$ Collaborative and social computing; Please use the 2012 Classifiers and see this link to embed them in the text: https://dl.acm.org/ccs/ccs_flat.cfm

\section{INTRODUCTION}

"See how relatable my captions were - stomach sucked in, strategic pose, pushed up boobs. I just want younger girls to know this isn't candid life, or cool or inspirational. It's contrived perfection made to get attention." - Essana O’Neill, 2015

Essana O'Neill was a micro-celebrity with over 600,000 followers who quit Instagram in 2015. She deleted much of her

Permission to make digital or hard copies of all or part of this work for personal or classroom use is granted without fee provided that copies are not made or distributed for profit or commercial advantage and that copies bear this notice and the full citation on the first page. Copyrights for components of this work owned by others than ACM must be honored. Abstracting with credit is permitted. To copy otherwise, or republish, to post on servers or to redistribute to lists, requires prior specific permission and/or a fee. Request permissions from permissions@acm.org.

CHI '20, April 25-30, 2020, Honolulu, HI, USA.

(C) 2020 Association of Computing Machinery.

ACM ISBN 978-1-4503-6708-0/20/04 ...\$15.00.

http://dx.doi.org/10.1145/3313831.3376424

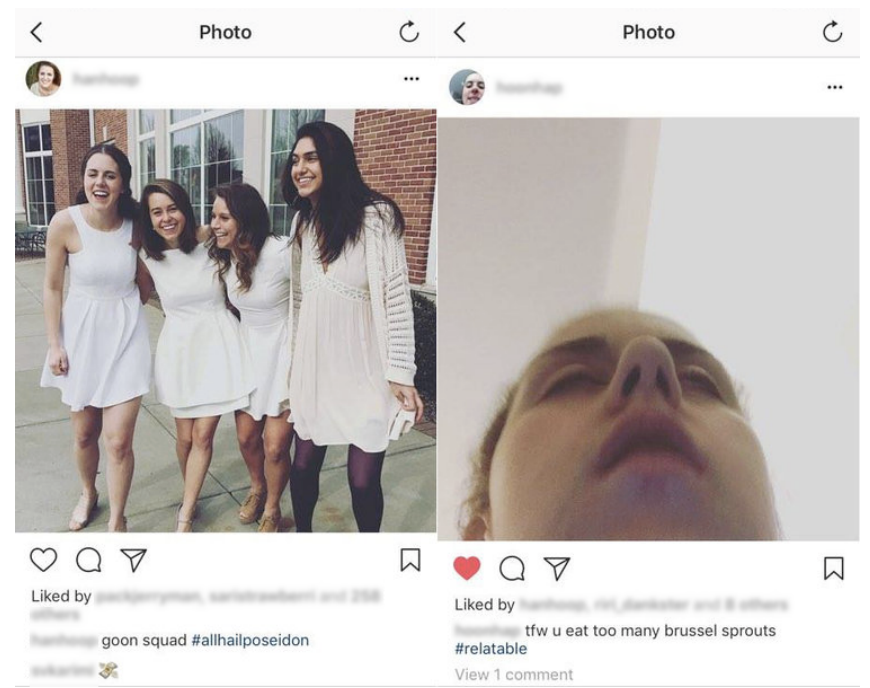

Figure 1. Two example photos from the same user's rinsta (left) and finsta (right) exemplify common differences in the two types of accounts. We obtained permission from the owner to reprint these images.

content and re-captioned the rest to draw attention to the unbearable pressure of the performance and the "fake intimacy" involved [22]. Many young people, and in particular young women [45], experience similar social pressures. These users have responded by reconfiguring the Instagram platform, leveraging the platform itself to create something novel and distinct, replete with a new name: finsta.

Finstas are often private Instagram accounts with a small follower list of close friends, and feature content that is ugly, silly, vulnerable, or otherwise unacceptable on the user's primary public account or rinsta $^{1}$ (Figure 1) [2, 19]. Finsta is defined at the intersection of the Instagram platform as technological artifact and users' socially constructed expectations, norms, and values. In creating finsta, users have carved out smaller, more intimate spaces within a larger sociotechnical system to express an alternative self with a trusted group of close friends. Following Lucy Suchman [59] we term this process an intimate reconfiguration.

\footnotetext{
${ }^{1}$ The term "rinsta" is a portmanteau of "real" and "Instagram." We use "finsta" to refer to users' secondary accounts; "rinsta" for their primary accounts, and "Instagram" for the platform itself.
} 
We sought to learn how and why people use finsta and its impact on their well-being and relationships. We conducted a qualitative study which took two main approaches: we asked about finsta directly in interviews and we analyzed existing discussions about finsta on video blogs. First, we interviewed ten finsta users and asked them to reflect on their finsta account, how they decided to create it, and what they use it for. Second, we sought to find people where they are by analyzing native formats of information sharing. We analyzed YouTube videos where people discuss their finsta through a popular type of video titled "exposing my finsta." In these videos, users display content from their finsta and discuss it.

We found that users put a lot of effort into and adopt specific practices to manage their audience on finsta in order to create a space where they can share an alternative self with their close friends. They use their finsta to share very different content than they would on their rinsta, including ugly, silly, introspective, and vulnerable content. Many users expressed gaining meaningful community support and experiencing therapeutic benefits from this outlet. We identify three key types of intimate reconfiguration on finsta that make this possible: 1) an unserious, messy self image in place of presenting an idealized self; 2) deep engagements with close friends in place of atomic, fast, and superficial interactions such as likes; 3 ) vulnerability and disclosing negative emotions in place of the expectation of a constantly positive and upbeat performance.

We argue that in creating new norms, expectations, and cultures, intimate reconfigurations provide relief from dominant social pressures on the platform and in society more broadly and provide the opportunity for alternative social configurations to form [25]. We situate finsta in a broader cultural moment shaped by neoliberalism to better understand the pressure young people face to present a perfect self brand on social media and their strategies to push back [45]. We also draw attention to the limitations and complexities of this strategy, which has great potential but is not immune from imposing its own pressures and expectations on users.

\section{RELATED WORK}

In this paper we rely on three main areas of related research: performance and self presentation online; secondary social media accounts, sensitive disclosure, and social support; and theoretical foundations from feminist science and technology studies that explain how capacities for action are configured at the interface of people and technology.

\section{Performance and Self Presentation Online}

Erving Goffman proposed a conception of everyday life as a theatrical performance. In Goffman's view, individuals take on roles and perform social interactions as if life were theater, attempting to keep their performances tailored to each specific audience with which they interact and social settings through which they move [27]. Of particular relevance to this work is the concept of a "front stage" and "back stage"-Goffman describes that individuals act differently when they are in view of their audience and must adhere to relevant norms and modes of behavior for the purposes of impression management, as opposed to their behaviors in more private settings hidden from audience view.

Impression management is made more challenging online because people have to grapple with social contexts that have different norms and expectations in the same place; a concept known as context collapse [10, 43]. Hogan argues that in these cases people limit their self-presentation to the lowest common denominator of what is acceptable to the people who may view the content [32]. Markham has argued that the Goffmanian conception of dramaturgy is further complicated in the digital era, when different (even past and present) versions of the self can co-exist in close proximity, collapsing the audience separation on which impression management relies [41].

Zhao et al. painted a multi-faceted picture of people's experiences of social media by relying on both Goffman's theatrical metaphor as well as Hogan's exhibition approach [32, 67]. They argued that people not only use social media as a stage to perform, but also as an exhibition of who they are, and as a personal archive or diary for themselves [32]. These overlapping regions are sometimes in tension with each other, in particular past public performances, although valuable as personal archives, may no longer be appropriate for public display. While this extension of the Goffmanian performance to three regions-performance, exhibition, and personal archive-is a helpful conceptual tool, it does not fully encapsulate secondary accounts such as finsta, which do not fall neatly into one of those three regions. Instead, we found that finstas combine aspects of all three but for a different purpose and audience.

The dominant culture on social media sites dictates that people are expected to present a positive image of themselves [22, 42]. The expectation for a positive self-presentation can be problematic. Research finds that when browsing other people's polished photos on Facebook, users may assume that those photos are representative of how the people in them actually live which makes them compare those images to their own lives and conclude that others live a happier life [16]. Social comparison on Facebook, Instagram, and Snapchat can lead to feelings of depression and loneliness $[33,66]$.

People also use social media platforms to express a range of emotions [4]. Emotion sharing is beneficial for well-being, and also helps people build social ties through shared empathy. [53]. However, it is difficult to share emotions that construct a negative self-image. Waterloo et al. find that the expression of positive emotions is perceived as more appropriate than negative emotions across a variety of platforms [64], and users of Instagram focus on positivity much more than on the other social media platforms [56]. People find it hard to reveal negative emotions, especially those related to stigma [3]. They adopt multiple strategies to cope with the situation, such as staying anonymous $[18,37]$, using private chat channels such as email [24], and creating secondary accounts [42].

\section{Secondary Accounts, Disclosure, and Social Support}

Many social media sites require users to present themselves within a rigid profile structure where they are expected to have one account as the "real" and "authentic" self [29, 42]. This requirement has existed since social networks first gained 
widespread popularity, with sites going as far as to purge accounts deemed "inauthentic" as Friendster did in 2003 [47]. Facebook also routinely removes accounts that they deem "inauthentic" and gives users the tools to report such accounts [29]. These policies are often at odds with many users' needs, conflict with their self-presentation strategies, and diminish user agency [42].

In response, some people create secondary accounts. Early secondary accounts on Friendster, called "Fakesters," were used for identity play $[11,12,42]$. More recently, finstas are used by emerging adults (ages 18-24) in response to the possible monitoring of their social media by family and potential employers [21]. Finally, people have long used similar strategies to separate professional and personal relationships in their heterogeneous social networks [20].

Allowing users space to express and separate multiple identities online is especially important for people whose selfpresentations may be stigmatized, costly, or even threaten their safety $[4,29,43]$. For instance, reconstructing online identity is viewed as a rite of passage for transgender social media users and users' management of multiple online identities during transition is important in facilitating life transitions [28]. Disclosing sensitive information online can be an effective way to gain social support. For instance, Instagram users can gain social support after disclosing negative emotions or issues of mental health, both because of the psychological benefits of image-based disclosure and community norms that result in positive engagement in response to such disclosure [4]. Finstas can also be an outlet for emotional catharsis in a safe space [44].

Social media has been shown to have both positive and negative effects on young people's affective well-being across different dimensions of use [65]. For instance, self-expression can be a means for affirmation, or concern about others' judgements. Similarly, interactions with others can create closeness, or contribute to feelings of disconnection. Researchers have argued that what influences people's positive or negative experiences on social media is their individual practices [39]. But individual practices are complicated by what actions are possible given our socio-material conditions and where agency lies. These are central concerns of feminist theory and science and technology studies.

\section{Feminist Reconfigurations}

In this work we draw on science and technology studies (STS) and feminist theory to understand how capacities for action are configured (and reconfigured) in social platforms $[1,59]$.

One of the issues at stake here is where agency lies. Following this literature, we move beyond studying finsta as purely technological or studying finsta users as autonomous individuals living in a world of separate things [59]. Instead, we identify agency, or our capacity for action, as configured in our dynamic relations to other people and things [1]. In other words, we study the whole socio-technical assemblage and ask how all elements including the technological platform, people, and social norms expand or limit the capacities for action.
In our study of finsta, we pay particular attention to how finsta came to be, or how it was designed. Aanestad argues that when changes to existing socio-technical arrangements are needed, people do the "in situ work of design in configuration," or the ongoing work of design that takes place in practice rather than in a lab [59]. Similarly, we find that finsta is created as on ongoing reconfiguration of the Instagram platform by finsta users.

\section{STUDY DESIGN}

Our goal in this research is to understand how people learn what finsta is, why they create a finsta, what they use it for, and what effects it has on them. To answer these research questions we conducted a qualitative study.

\section{Gathering Data}

We gathered data in two main ways: directly in interviews, and indirectly through content analysis of video blogs about finsta. These two sources of data are complementary: In interviews we were able to ask people to reflect on their finsta and probe them to dig deeper into their motivations and experiences by asking questions. Analyzing native formats of information sharing on the other hand gave us a window into how people talk about their content with others without the perceived presence of researchers. For instance, in video blogs we found instances of bullying on finsta that we expect people would have hidden from us in interviews if they existed. Another motivation for these two sources was our ethical obligations. Because of the sensitive nature of finsta, our university's Institutional Review Board (IRB) did not give us permission to view the photos of finsta users we interviewed. Therefore, video bloggers who had voluntarily shared their finsta photos on YouTube, a public platform, gave us the opportunity to view and analyze finsta content.

\section{Interviews with finsta users}

We recruited 10 participants through a combination of purposeful and snowball sampling [54]. Initially, we posted on our social media accounts a brief recruitment survey for people who have a finsta account. We invited those who owned at least one finsta account and were at least 18 years old to an hour-long in-person interview near a west coast campus town in the US, or through a remote video call. At the end of each interview, we asked participants if they know anyone who actively uses or has used finsta and would be open to participating in the study. If yes, we reached out and invited those potential participants.

We conducted the interviews from June to July 2019. The interviews were semi-structured and we paid participants a $\$ 25.00$ gift card for one hour of their time. We asked questions meant to probe the participants' usage of, and motivation for creating a finsta account. We did not directly look at the participants' finsta accounts and only relied on the verbal description of their account and posts. We recorded the interview as per the participants' approval, and later anonymized and transcribed it for analysis. Except for one participant who owned 3 different finsta accounts, all other participants owned one account that they called a finsta. Our participants were 21.6 years old on average with an age range from 18 to 26 . 
There were $60 \%$ Asian, 20\% Caucasian, 10\% Hispanic, and $10 \%$ other. The majority of our sample ( 9 out of 10 ) identified as women. This is consistent with prior work that has found the population of finsta users is largely composed of women in their late teens to twenties [19]. We refer to the interview participants as P1-P10.

\section{"Exposing my finsta" video blogs on YouTube}

In order to gain further insight into the types of content people post on finsta in an indirect way, we searched for "exposing my finsta" in the YouTube search bar using an incognito browser. "Exposing my finsta" videos have become a popular category of videos in recent years, particularly among YouTube microcelebrities. In these videos bloggers display screenshots of their finsta posts, often accompanied with a commentary discussing why they posted that content. They generally keep their finsta handle private. While these micro-celebrities are likely not representative of the larger user population of finsta, we found that their motivation for having a finsta account and their content was very much in line with that of regular users we had interviewed.

We selected the 10 most recent videos on YouTube that included the blogger's commentary on their finsta in August 2019. These videos had 408,000 views on average, and their creators had 146,000 subscribers on average. We analyzed the transcription of these videos. In order to learn about the types of content that are often shared on finsta we also captured and analyzed 224 screenshots of content from their finsta accounts that they showed in their videos. Although these videos are created for public consumption and are fully accessible to any person on the web we have not included any identifiable information in this paper. We refer to the YouTube video bloggers as Y1-Y10 throughout the paper.

\section{Ethical considerations}

Most finsta accounts are private, and they often contain photos and captions that are sensitive or personal to the user and others who are featured in them. We tailored our methods to protect their privacy by relying on participants' verbal description of their account during interviews rather than viewing any of that content directly, and by not reproducing any identifiable information from the YouTube videos. For instance in the process of our research on YouTube we found a host of videos exposing other peoples' finstas, we did not view or analyze those videos. There are many ways to respect and preserve the privacy of these intimate online spaces; we do not suggest that all future work must take exactly these measures, but we do propose that future researchers in this space be mindful and intentional in their orientation towards user privacy.

\section{Limitations and opportunities for future work}

Our data gathering method biases our sample to finsta users we had ready access to via our social networks and people who post video blogs on YouTube. Additionally, some video bloggers selectively exposed a portion of the posts on their finsta. While we believe the finsta content they shared is typical of finsta content because it maps onto what our interview participants also told us, we may not have an accurate proportion of each type of content. We also expect that "exposing finsta" videos have a performative nature, and the content they selected to expose may be catered to their audience. In future work we will study a larger sample size and use survey and quantitative methods to learn how widespread our findings are as well as what the properties of the finsta social network are.

\section{Analyzing Data}

The data we analyzed was made up of: 1) transcripts of the in-person interviews, 2) transcripts of YouTube videos, and 3) screenshots of finsta posts that they shared in their videos. We conducted interpretive qualitative analysis for the text data (1 and 2) [46]. We started with open coding in two phases [15]. In the first phase, we coded the transcripts on a line-by-line basis so that our code would reflect our data as closely as possible. Examples of such codes include "relationship maintenance" and "record memory." In the second phase, we focused on synthesizing the resulting codes from the first phase to extract higher level themes that our data represents. Examples of these higher level categories include "motivations", "emotions" and "content." Besides the analysis of text data, we used the screenshots of finsta posts as supplementary data to the transcripts and developed codes for them in a similar fashion. The codes for screenshots described the content of posts for instance: "crying selfie" and "photo irrelevant to caption."

Our process of coding was iterative; we continuously reviewed both our categories and data in order to find patterns within our data and synthesize our findings into resulting high level themes. During the process, We found that participants frequently referred to rinsta to contrast and contextualize their finsta usage. As a result, we added tags to our interpretive data analysis to differentiate when participants were referring to "finsta" or "rinsta." In comparing those quotes we found participants reported using features of the platform to carve out a space for finsta when rinsta could not serve their needs, which we detailed in the next section.

\section{FINSTA}

We describe our findings on finsta in three sections: setting the stage, performing finsta, and communal and personal uses of finsta. In the first section we describe the necessary groundwork for having a finsta including the process of creating an account, how users manage their audience, and how they create a safe space for the presentation of an alternative self. In the second section, we focus on users' content and interpersonal interactions on their finsta accounts, in particular their aesthetics and emotional affect, and how these differ from their rinstas. Finally, we describe the communal and personal uses that people reported their finstas have for them.

During the interviews, we found participants constantly compare their finsta to their rinsta. Before delving into findings regarding users' finstas, we summarize the users' rinstas usage at the beginning of each section, and also refer to rinsta later when participants made comparisons.

\section{Setting the Stage}

In this section we first describe finsta as a safe space for an alternative self and then discuss how people create that space. 
a

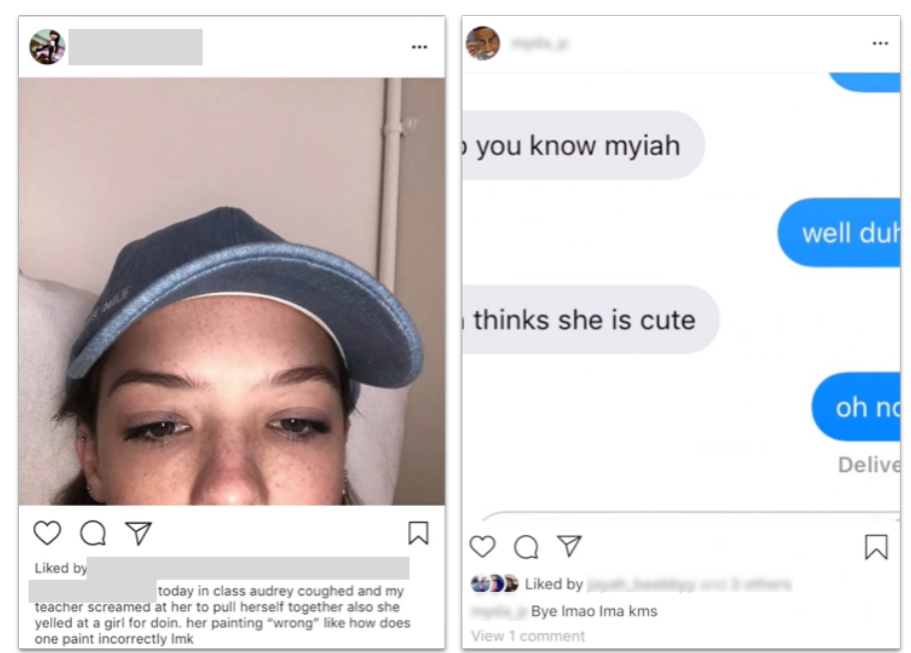

C

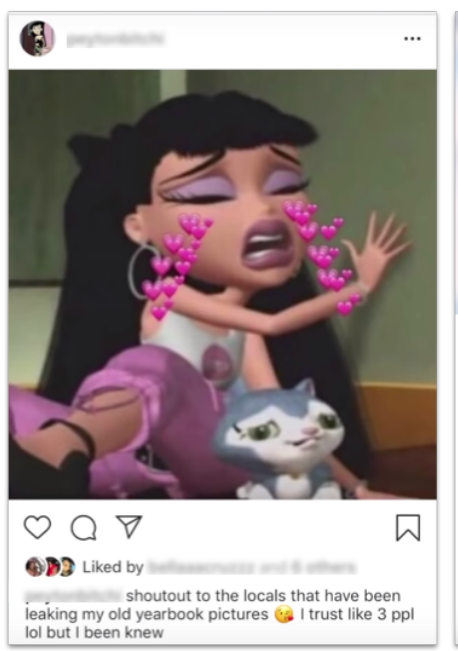

d

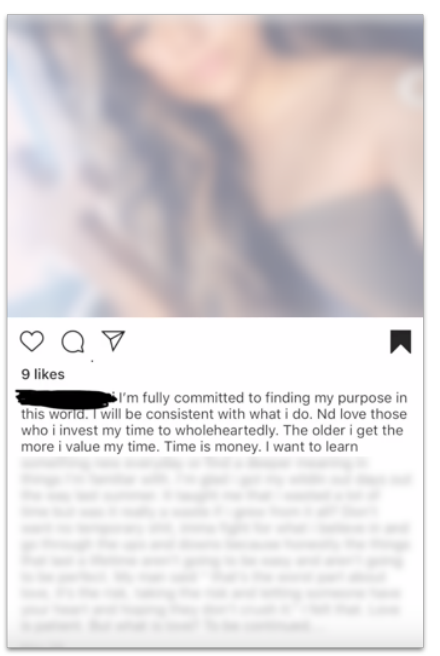

Figure 2. Example finsta posts that video bloggers on YouTube have shared under the title "exposing my finsta." (a) A low-quality selfie. (b) A user posted a cropped screenshot of a text conversation with a friend. (c) A user complained about people leaking her yearbook pictures with sarcastic caption and a meme picture. (d) A user posted a selfie and wrote a long caption to reflect on her purpose in the world.

Finsta is a safe space for presentation of an alternate self All of our participants had a rinsta account prior to the creation of their finsta account. We found that their rinsta accounts are often public. For those who had private accounts, they were open to accepting follow requests indiscriminately, including from friends, acquaintances, family, and strangers. P1 described her rinsta audience as "unregulated." Content is accordingly shareable with a wide audience: "they're pictures that I would be comfortable with anyone seeing" (P10).

In contrast, most participants describe finsta as a place to present an alternative self. Finsta is seen as a place to post "any other things" not captured in public facing social media channels. P6 said, "I feel like for me, it's a social media site that has everything that I wouldn't put on any other social media." P9 describes it as an outlet free from the usual pressures associated with an online presence: "it's not branded as you, necessarily, and it's not publicly linked to your identity. I think it's also an account where you can post whatever you want." On finsta, users were not worried about being judged [65]: "A lot of people will need that space (finsta) not to be judged. They have either very difficult or oppressive social environments either from family or from strange private groups, so for them that's like their creative outlet so to speak" (P1).

While participants described their self-presentation on finsta as "alternative," most of them do not see it as more real or accurate. P4 instead describes it as a place to "showcase more of my personality." P10 summarized,"Neither of them [is] a completely accurate representation, and together maybe they are more accurate than either one alone."

Next, we report users' practices around setting the stage for their finsta accounts - creating a finsta, making a profile, and choosing followers.

\section{Motivations for account creation}

We found that the finsta phenomenon spreads through interpersonal networks; most participants said they had created a finsta account after seeing their friends' finstas. Users also reported liking the idea of a less polished account shared with close friends. One participant summarized, "Part of it was because it seemed like what everyone else was doing at the time. So, I hopped on the bandwagon. But, I also really liked the way that I could drop any pretenses that I have on my personal Instagram account " (P4).

The platform itself and its affordances also factored into some participants' decisions to make a finsta. P8 compared Instagram to Facebook, explaining, "there is something that's very simple about [Instagram], where it's like, oh, add account, make a username, it's out in the wild. If I wanted to make like a fake Facebook that would be so much work." A real name policy and review of every new account are both central to Facebook's account creation policy [30]. In contrast, Instagram allows users to be simultaneously logged into multiple accounts and easily switch between them, a feature that was only added after the rising popularity of secondary accounts [49].

\section{Creating a finsta profile}

After deciding to make an account, the next step is profile creation. We asked participants to describe their profiles, including the profile photo, username, and optional short bio. Participants reported using somewhat obfuscated usernames and photos: "I chose a really ugly photo of me and zoomed in so you couldn't tell it was me" (P4). Users suggested that straddling the line around identifiability allowed them to connect with friends who know them personally, without broadcasting their identity to strangers; P6 explained, "I am identifiable, but I'm private also, and it's also not like you would know exactly who I was unless you actually knew me, I think" (P6). Several 
participants mentioned using a nickname rather than either a real or entirely fake username.

\section{Building an audience}

In contrast to their rinstas, users reported exerting a much higher level of intentional control over the audience on their finstas: "finsta was kind like of a way that I could put controls on who sees what, or who's even allowed to know what"(P1). Nine of the ten interview participants had private finsta accounts, visible only to their followers. Even with this level of care, P2 reported lingering privacy concerns, noting that screenshots of her finsta posts could circulate.

Eight of ten participants reported restricting followers to those they perceived as close friends. P5 has 60 followers on finsta, and she described her followers as: "they're people who I've definitely all met in person and have known for at least a year, and know them on a rather personal basis. I've had multiple conversations with them, feel comfortable telling them a lot of stuff. I would say good friends, like my inner circle." Some participants explicitly mentioned trust as the criteria for allowing someone to follow their finstas: "I have to trust you as a person and I've probably already talked to you about some of the stuff that I share on my finsta before I let you into my finsta" (P4). A notable exception to this rule was made for aspirational connections or people the user wanted to build a friendship or romantic relationship with, despite not having one yet: "I really want to be friends with this person but we're like familiar strangers so I let them follow me" (P3).

Two participants were exceptions to this norm. P7's criteria was looser: "I just accepted them because if they wanted to follow, I didn't really care that much" (P7). And P8 has a public account: "If you find it, like, good for you. But I'm still going to post the stuff I want to post" (P8). However, P7 and P8 said their followers are still mainly their close friends. P6 reported having a looser criteria initially, but she made her audience narrower to close friend later, because her initiallyloose criteria led her to stop posting on the finsta: "I really didn't know these people that well and they had just found me ... I was putting that much personal or dramatic information but I didn't want to really tell them"(P6).

Most of the participants were comfortable with more distant connections being aware of the existence of their finsta, but if such people sent a follow request, it would be denied: "I don't care if people know that I have a finsta, but I wouldn't accept them" (P10). They considered it a standard practice: "I respectfully decline their requests and just hope that they forget about it" (P4). In contrast, P1 was concerned about how request rejections would be received, and proactively blocked accounts to avoid her finsta being discovered by real-life acquaintances whom she did not want to know about her account: "it was more like a security measure, because if [non-follower acquaintances] even were aware of [my finsta]... it's like, they'll confront you, and I didn't want that kind of pressure."

All participants reported that their followers often came from disparate social circles or parts of their lives and might not know each other. One participant reported that this resulted in cross-social group interactions on her finsta: "It's fun to see sometimes people from different parts of my life will come together and have a conversation in the comments of my finsta or build off of each other" (P10).

Participants described the choosing of followers as a continuous process, in which incoming follower requests are considered, and current followers are also reviewed. P4, P5 and P10 described the removal of their followers as relatively commonplace, not in response to a particular incident but rather as a reaction to ebbing of intimacy and closeness over the normal course of a friendship: "The relationship could have soured, although I don't think that's really ever happened to me. More so, it's the relationship has changed in that we haven't put in the time to keep up" (P4).

When we asked participants about which accounts they follow from their finstas, most participants described their finsta follow relationships as reciprocal, reporting that the followers they accepted on their finsta account were the same accounts they chose to follow. Some participants expressed that they would only follow friends' finsta accounts from their finstas.

Some participants also reported using their finstas to follow accounts they would not want to follow from their rinstas, either to avoid publicly linking their identities to those accounts, or to quarantine certain content from their main feed: "I feel like I follow meme accounts, or like accounts that... post a lot. Because I don't really check my feed on my Finsta as much... it's like, accounts that I want to follow or show support to, but I actually might not want to see all the time"(P8). P3 mentioned that one of her friends uses finsta exclusively for following accounts, and never for posting their own content.

\section{Performing Finsta}

In this section we describe the types of performances common on finsta [27]. Similar to the last section we begin by with a short description of rinsta.

\section{Rinstas are often polished and self-serious.}

Participants experience pressure to present a polished, idealized version of themselves on their rinstas, resulting in selffocused and positive-affect content. P5 said, "I think of real Instagram [rinsta] as a way to portray your best self and everything that's going right with your life, and all your skills and talents and everything happy." Several users specifically mentioned happiness and positivity as the dominant emotions in their rinsta content. P4 used his finsta as an analogy: "My rinsta is kind of like my finsta, but almost the best possible version, where it's as if I had no problems."

Many participants reported spending significant time selecting, editing, and curating rinsta content. $\mathrm{P} 4$ admits that he spends a lot of time thinking about what to post, and tries to keep all his rinsta posts visually consistent: "I have a whole process for editing my photos and stuff ... there are certain filters there, and settings, that I find aesthetically pleasing." Relatedly, participants reported seeking validation on rinsta in the form of likes and followers.

\section{Finstas are unfiltered, silly, and sometimes NSFW.}

On finsta, users post content that they perceived as ugly or low quality (as shown in Figure 2, image A) and did not meet 
their aesthetic standards for rinsta. Many participants echoed P10's sentiment that, "I spend some time editing photos on my rinsta and I spend zero time editing finsta posts" (P10). The expectation of low-quality, unfiltered content seemed to be perceived as a cultural norm to most participants. P4 described, "for my finsta, I don't edit anything. Sometimes, if I do edit it, it's almost in a way to accentuate how ugly something is, or how chaotic or how ... unfiltered, I guess, it might be.” P5 also described her content as "happy but messy," "like getting really, really drunk with friends, and it's really happy but it's not the most positive thing to share to the rest of the world."

Users also expressed sharing taboo or stigmatized content on their finstas, such as sexual, risqué, or otherwise "not safe for work" (NSFW) content. Twelve percent of images shown by YouTubers fell into this category. For instance content in which Y4 described herself as "half nude," and another featuring drugs that prompted her to say: "I feel like such a bad influence showing you guys these" (Y4). One of our interview participants expressed associating sexual content specifically with accounts belonging to her women friends: "a lot of people post, like, risque photos on there [...] that was, like, a major thing that certain people do-it wasn't everyone $[\ldots]$ it was primarily the women, I would say" (P2).

Participants reported using humor to signal a level of unseriousness that explicitly contrasted their finstas with their rinsta as a place for serious, carefully-presented, or polished content. One participant explained that her profile photo was, "a funny picture with me just rolling my eyes or something like that. Just something that would make it obvious to people [...] that it's a fake-not, like, a fake, but a funny outlet rather than any professional or real Instagram [account]" (P7). P5 posts about dancing on both finta and rinsta, but she noted how those were different: "on my finsta, it's me making a bad face while dancing and I'll make fun of that whereas on my Instagram [rinsta], it's prime dancing photos, photoshoot-level."

\section{On finsta people reflect and share difficult emotions.}

Much more than their rinsta, we found that people used their finsta to express negative emotions. 29\% of posts from YouTubers discussed difficult topics such as failure or disappointment, or other related emotional content such as frustration, sadness, or anger. One YouTuber explained, "a lot of my stories is me in a fucking pissed off mood" (Y9), while others showed photos they had posted while upset, including selfies of themselves crying: "I took a selfie of myself while I was crying, and I pretty much said just that life was shitty at that moment" (P5).

Several participants (P3, P4, P5, P7) specifically described their finstas as a place for venting. P7 described, "my last post was more of a venting post where I was talking about just how there's some challenging things are happening in my life." Another interviewee described, "[On finsta] I talk about bad grades, bad tests, bad relationships and different problems that I have in relationships" (P5). In contrast, sharing difficult emotions is not common on rinsta. P5 said: "I would never in a million years say that I am depressed at a given time [on rinsta]. I have 800 followers on my real Instagram and most of them would be pretty uncomfortable seeing that, and I feel like
I would be uncomfortable with them seeing it [... my finsta is] a place to focus on all of the negative things."

Participants found this outlet for negative emotions valuable, particularly in difficult times. P3 once deleted both her finsta and rinsta to limit her social media use, but after her friend experienced a tragedy, she restored her finsta: "I was feeling very lonely ... so it was something emotional that happened in my life and that was what made me get on social media again."

While in ugly or low-quality photos humor signals a level of unseriousness, humor was also commonly used to mitigate the gravity of negative emotions. We fount that $13 \%$ of the posts in the YouTube screenshots we analyzed used humor to describe difficult topics or mitigate negative emotions. In Figure 2, image $\mathrm{C}$ shows an example post where a YouTuber complained about people leaking her yearbook pictures with a sarcastic caption and a meme photo, making light of an incident that had been very upsetting to her. P7 posted on her finsta when she attended her friend's wedding: "I have a really random face, I look like I'm scared of something, and it was at her wedding shower, and so I was like, 'when all of your friends are getting married and you're like, single af' or something. So again, venting about that."

Several participants also use finsta for reflective posts, where they share their thoughts at the moment. P6 said, "I would just write, not even a reflection, it's not really planned. It's how I'm feeling in that moment." As the Instagram platform is focused on sharing images, one striking feature unique to finstas was the prevalence of text-based content. This includes posting reflective texts, tweets or screenshots of SMS conversationsfor example, in Figure 2's Image B, a user posted a cropped screenshot of a text conversation with a friend in which she learned her crush was interested in someone else. Users also described posting "random" photos as a vehicle for text content in the captions. P5 said,"some of them, the photo doesn't really have to do with what I'm talking about. One is a photo of a soft serve I was eating, but the caption is me talking about how I'm really mad at my mom." As is shown in Figure 2's image D, a Youtuber reflected on her "purpose in the world" with a selfie. In YouTube screenshots, we found that $11 \%$ of posts had unrelated text and image.

While users post about reflective thoughts and negative emotions, several participants (P3, P5, P6, P7) said that they did not post about those kinds of content on finsta initially. Instead, they describe their use of finstas changing over time from posting ugly, silly content to spaces where they posted reflective or emotional things: "[Before] the content was [...] funny things that I wanted my friends to see, but eventually it became more of like me reflecting about like more serious things, or if I couldn't talk to my friends about something, I would like post about it and like hope they would read it" (P3).

\section{Personal and Communal Uses of Finsta}

Finally, we found that people found finsta as a safe space where they could vent and get emotional support and interact with others in ways that they found more meaningful and genuine. 
People use finsta to vent and get emotional support.

Several participants told us that they find finsta helps them to vent negative feelings and get emotional support. P5 said that writing about the experience allows her to "share the mess inside my head and put it into words and a visual" (P5).

P3 reported using finsta to passively reach out to friends, especially around sensitive issues that are hard to discuss in person: "If I couldn't talk to my friends about something, I would post about it [on finsta] and hope they would read it." P5 describes how talking about stigma on finsta works as therapy: "It's a way for people to talk about mental health and things that have a stigma [...] It's a way to talk about drugs and alcohol and sex [...] I've sometimes compared it to therapy, because you're giving voice to things that bother you, and it's a place where you can share it with several friends or just the people you feel will understand you."

The greater depth of interactions and the audience of close friends combined to garner participants a sense of emotional support and psychological safety from their finsta accounts. P5 described how she benefits from viewing others posts and receiving support besides posting: "It reminds you that there's other people going through things [...] and they can tell you, 'It will be okay' and 'This is how I got through it.' Sometimes people will share coping methods or really it's just a way to talk it out."

Notably, users reported providing the same support to their friends in return: "I always see the finsta as another opportunity to look out for my friends. If they post something that's really concerning to me, I will reach out to them in person" (P4). The shared empathy and mutual emotional support strengthens relationships with those around them: "I think it affects how I'm perceived by my friends, but I also think that it's important for them to understand what I'm going through. I think it builds empathy and sympathy" (P5).

\section{Interactions on finsta are perceived as meaningful and gen-} uine.

Participants generally considered their interaction with the audience on finsta as more meaningful and genuine compared to their rinsta. Several participants (P3, P4, P5, P6, P10) reported that higher counts of likes and followers were valuable on rinsta, but that these interactions at times felt superficial. P3 said, "By pure volume, I interact with my friends more on my rinsta, but in depth of interaction, I think my finsta."

P4 explained why she thinks interactions on finsta are more genuine: "Because they [finstas] are private accounts and because these are people that I've screened, if they comment or if they make a joke with me or stuff like that, I feel it's more genuine. Or, I think it's genuinely them that's speaking and not maybe a public facing version of them."

\section{INTIMATE RECONFIGURATIONS}

In the previous section we described how people use finsta: the work of creating an account, users' performances on those accounts, and the impact it has on them. Finsta-the combination of Instagram's platform and the human norms, expectations, and behaviors that comprise it-is a sociotechnical assemblage residing on Instagram's infrastructure, but fundamentally different in its capacities for action from rinsta [59]. We term this repurposing of the platform to create smaller, more intimate spaces for close friends an intimate reconfiguration. Next we detail three intimate reconfigurations that are central to finsta, and then situate the reconfiguration process in feminist science and technology studies.

We found three key ways that finsta users have reconfigured Instagram. These include emphasizing: (1) unseriousness and imperfection in place of an idealized self and personal branding. (2) lengthy, deep engagements in place of instant, superficial interactions (e.g. Likes) and (3) vulnerability in place of obligate positivity. Below we will describe each configuration in more detail.

\section{Idealized Self $\Rightarrow$ Unserious Messiness}

In response to rinsta norms of polished aesthetics and cultivating a personal brand, users' finsta accounts reconfigure these in favor of unseriousness, humour, and imperfection.

Youth face social pressures to maintain an image of perfection online for many reasons, including pressure from peers and the potential for their online content to impact college admissions and current and future employment $[10,52]$. At the same time as social media has increased in ubiquity, the lines between the personal and the professional have blurred, leading many users to cultivate a more professional identity online [10]. One participant explained that she keeps her professional and personal social media separate, and would be concerned for her public image if her finsta content were made public:"It's very, again, very like a funny, random venting outlet and I don't think that would be a really good image that I necessarily want anyone in my professional network who doesn't really know me see"(P7).

The pressure for polished aesthetics on rinsta can be understood within the broader context of the intensification of beauty pressures on young women [22]. Women must mold and shape their bodies to emulate societal ideals of youth, heterosexuality, ability, whiteness, and thinness [8]. A host of beauty apps and filters exist that encourage women to surveil themselves through a "pedagogy of defect" and to make adjustments [7]. Under these conditions, creating space for imperfection through finsta accounts temporarily restrains those pressures and even pokes fun at them with ugly selfies.

\section{Superficial Interactions $\Rightarrow$ Deep Engagements}

On finsta, users have reconfigured the norm of what they viewed as superficial, fast-paced interactions such as likes to lengthier, deeper engagements. This often took the form of lengthy captions on images and long comments: "[My] regular Instagram account gets very generic kind of comments. If I ever get comments it's usually like a 'You look so good!' Or heart emojis kind of things. Very basic [...], whereas finsta comments, I feel like people will talk a bit more personally and relate to it more"(P5).

Research has shown that people grow closer to others online when they engage via composed pieces such as comments and posts more so than one-click actions such as Likes [14]. 
Dominant forms of interaction on social media have been characterized as short, episodic, and transient; limiting peoples' ability to engage in lengthy, thoughtful reflection [62]. Finsta users have changed that norm to one where long and deep engagements are expected. This is possible because of the limited size of the audience and the fact that a person's finsta followers are usually close friends.

\section{Obligate Positivity $\Rightarrow$ Vulnerability}

Finally, we found that finsta users reconfigured the requirement to present a positive, upbeat persona to space where negativitiy, complaining, and vulnerability were acceptable.

On my rinsta, I'd only post really happy things, and specifically, tailored happy things. (P4)

Researchers have argued that young women in particular are increasingly required to invest emotional or affective labor in producing selves that are agreeable for others [38]. We also found references to that labor in our research.

My Instagram is very curated [...] It's always really good photos of me, really good dance photos of me, or with friends when we're all dressed up or going out or styled [...] A lot of them are DSLR kind of photos, highly-edited, great color balance and all of that. (P5)

On the other hand, finstas provide an escape from those pressures to present an alternative side:

I think it was just a show like a different side of me that I feel that it's like really hard for me to verbalize things emotionally sometimes or open up to my friends in a certain way. So I definitely leverage my posts so that like I could show a different side of myself that is a little bit more vulnerable and has a little bit more depth. (P3)

Therefore people use finstas as an opportunity for performances that are not necessarily positive or upbeat but may be silly, sad, or vulnerable.

\section{Who Reconfigures Technology and How?}

Feminist science and technology studies (STS) scholars have long studied how agency, or capacity for action, is shaped at the intersection of people, technology, and practice $[1,10,35$, $58,59]$. Finsta is one example of users finding creative ways to gain (or regain) agency by leveraging the existing tools available to them. In some cases, these reconfigurations can result in concrete changes to the technological infrastructure. For example, in 2016, Instagram rolled out a highly requested feature allowing users to create and navigate between multiple accounts on the same device. Speculation among the user base and the media suggested that this represented a move by the company to cater to a younger population of users, among whom the practice of having a finsta was common [51]. This feature was unexpected, given that Facebook, Instagram's parent company, has held a strict "real name" policy, routinely removing "inauthentic," pseudonymous, or duplicate accounts from the Facebook platform [30].

The social norms and technical features that comprise finsta point to its users as designers of the sociotechnical assemblage.
This is counter to the dominant view of design, which separates centers of innovation from peripheries where the fruits of that innovation are consumed $[35,36]$. This view overlooks everyday design by users [63]. In online social spaces in particular, experiences are shaped more so by interactions with other actors (e.g. norms) than by the technology itself $[50,12]$. For instance, boyd details the myriad ways in which youth find innovative ways to achieve social connections while maintaining privacy online [10]. Gender is central to recognizing finsta users, who are mostly young women, as designers. This argument stands in contrast to views of sociotechnical design as the sole province of Silicon Valley entrepreneurs, designers, and engineers who are predominantly men $[1,34,59,60]$.

Beyond who does design, feminist STS teaches us to ask how design is done $[35,59]$. Suchman shifts our frame from the heroic designer of extraordinary new technologies to "ongoing, collective practices of sociomaterial configuration, and reconfiguration in use" $[57,59]$. This necessitates a recognition of the ongoing work of design that takes place in practice by users [1]. Following this view, finstas are enacted through everyday mundane and innovative acts such as using Instagram to create private accounts, manifesting community through following others, and setting norms around content sharing [10, 59]. Through this labor users are simultaneously using finsta, and shaping what finsta is.

Feminist theory directs our attention to the often taken-forgranted labors that sustain complex socio-technical assemblages such as rinsta or finsta [59]. Here we draw attention to two distinct types of labor: emotional and intimate labor. Emotional labor was first used to describe the work of regulating ones emotions in the context of service jobs [31]. For instance, a waitress has to express positive emotions to customers regardless of how they feel. Intimate labor or care work is the work of tending to the intimate needs of individuals such as health and hygiene maintenance, caring for loved ones, and sustaining social and emotional ties [9]. Based on our findings we argue that rinsta is sustained through the emotional labor of creating and maintaining an always upbeat, positive persona, while finsta relies more heavily on the intimate labor of caring for close friends.

\section{COMPLICATING FINSTA AS FEMINIST UTOPIA}

In this paper we have laid out a description of finsta as a user-designed reconfiguration of Instagram. In this section we draw attention to some of the possibilities it provides for reimagining our sociotechnical artifacts and our society, as well as a more cautionary critical take on the same.

\section{Countering Neoliberalism with Intimate Reconfigurations}

Finstas (and rinstas) have become popular at a distinctive cultural moment shaped by neoliberalism and postfeminism [22, 23]. Much has been written about neoliberalism in recent years (see [13]), yet it remains a complex, contested, and much debated term $[17,26,48]$. Most commonly neoliberalism involves the extension of market principles to all areas of life $[13,22,55]$. Some scholars have described it as a worldview that assumes individuals as self-concerned agents not members connected to a community; with competition as the 
defining characteristic of human relations. From this vantage point citizens are redefined as consumers and markets are the ideal means through which people can meet their needs [17, 48]. Others have related neoliberalism to a sense of insecurity, perpetual competition, and individual isolation in society [26]. As the dominant form of public on Instagram, rinsta is culturally shaped by neoliberalism [22, 42]. This is through the norms and cultures that people have developed on rinsta and amplified by the technical affordances of Instagram (e.g. emphasis on the numbers of likes and followers as currency).

In this view, finstas can be seen as counterpublics that offer a temporary suspension of the forces of dominant publics and explicitly articulate alternatives to them [5]. Fraser uses the term subaltern counterpublics to describe spaces such as women only voluntary associations where subordinate groups can gather and talk [25]. Mansbridge envisions "protected enclaves" where people can explore ideas in an environment of mutual encouragement [40]. For Fraser counterpublics offer the potential for issues that have been overlooked, purposefully ignored, or supressed by the dominant public to be brought forth and discussed. This allows subordinate groups to formulate alternative interpretations of their identities and interests [25]. For instance, early feminist counterpublics formulated and contested issues previously dismissed as concerns of the private sphere, such as domestic abuse.

Under neoliberalism, the 'perfect' has emerged as the horizon of expectation for young women [45]. Competition to constantly improve themselves toward a fictitious perfect life directs attention to individualized self-regulation and stifles the possibility of an expansive feminist movement [45]. In this context, finsta offers the possibility of constructing a space and language to practice imperfection, intimacy, and solidarity building. For Fraser the existence of multiple publics, and counterpublics in particular, are a necessary requirement for deliberation in society [25]. At the same time, she points out that the fact that counterpublics widen discourse and contestation does not necessarily mean that they are virtuous.

\section{Potential for Abuse}

While protection from dominant publics and less restrictive social norms offer many benefits particularly to subordinate groups, the conditions for social exclusivity, bullying, or harassment may emerge. While finsta users in our data never explicitly admitted to such behavior, some of the screenshots we analyzed nevertheless suggested that it occurs.

Further, as we observed in the data, the lifting of norms does not leave an empty vacuum behind - other standards and expectations fill that void, not all of which are desirable. In the interviews, participants described that finsta sets new expectations for them. As P6 puts it, "I feel like there still is some expectation for you to be funny, or for you to be down to earth [on finsta]." Some finsta users reported that they feel they are expected to be open, be funny, or even be imperfect on finsta. They also feel obliged to provide emotional support. P3 reflected, "When someone lets you into that side of them or that life, you do have to take up some of that you know relationship where you're confiding in them, they confide in you, et cetera $[\ldots]$ sometimes I have felt that way where I knew because it was her finsta, and because she was sharing it with only a few people, that I should say something."

It is also worth considering the impact on the audience. For example, the corollary to users' comfort in discussing negative emotions and weighty topics is audience exposure to such content, which may be emotionally exhausting, triggering, or merely unwanted. Two other related problems are peer pressure and content incriminating of oneself or others-regarding drugs and alcohol, one of our participants mentioned, "there's also a lot of substances put on there which isn't as big a deal in college, 'cause people are of age, but I know when people are younger, [they] are still posting things like that" (P2).

Finally, these intimate reconfigurations are by definition on the same platform as the original space whose norms, culture, community, or affordances were toxic enough to warrant the reconfiguration. We might ask, then, whether keeping finsta and other similar practices on these original platforms helps maintain the relevance and primacy of the platform itselfshoring up the very toxicity those reimaginings sought to avoid, in place of radically remaking entirely new spaces.

\section{Accountability and Expanding Frames}

"We are responsible for the world in which we live not because it is an arbitrary construction of our choosing, but because it is sedimented out of particular practices that we have a role in shaping." [6]

In this work we have taken a critical lens informed by feminist STS to understand finstas as intimate reconfigurations of the Instagram platform. We have discussed the possibilities of finstas as counterpublics that expand the space of discourse while complicating their actual effects in the real world. We argue that finstas are neither the embodiment of a feminist utopia, nor entirely counter-productive. Instead, we draw attention to how people have reconfigured finsta and the effects this particular reconfiguration has on them, "not that this will save us -but it might open our imaginations" [61].

\section{CONCLUSION}

Finstas give us a lens into the practice of intimate reconfigurations: the repurposing of an existing sociotechnical platform to subvert its function as a stage upon which users are constantly performing a palatable, presentable version of self, instead carving out a space of greater privacy and emotional support. By qualitatively analyzing the way ten interview participants and ten video bloggers describe their finsta accounts, we identified reconfiguring practices including presenting an unserious, messy image of self; valuing deep connections over a myriad of superficial interactions; and engaging in emotional vulnerability rather than obligate positivity. While imperfect, we argue that these reimaginings help users push back against dominant social forces, providing them with an intimate reprieve from the pressure to be constantly marketing oneself and presenting a coherent and palatable personal brand.

\section{ACKNOWLEDGEMENTS}

Special thanks to our participants. We would also like to thank Sofia Dewar, Schinria Islam, and Elizabeth Resor for their early work on this project. 


\section{REFERENCES}

[1] Margunn Aanestad. 2003. The camera as an actor design-in-use of telemedicine infrastructure in surgery. Computer Supported Cooperative Work (CSCW) 12, 1 (2003), 1-20.

[2] Miranda Abrashi. 2018. The fake account for the real self. (2018).

[3] Nazanin Andalibi, Oliver L Haimson, Munmun De Choudhury, and Andrea Forte. 2016. Understanding social media disclosures of sexual abuse through the lenses of support seeking and anonymity. In Proceedings of the 2016 CHI Conference on Human Factors in Computing Systems. ACM, 3906-3918.

[4] Nazanin Andalibi, Pinar Ozturk, and Andrea Forte. 2017. Sensitive self-disclosures, responses, and social support on instagram: the case of\# depression. In Proceedings of the 2017 ACM conference on computer supported cooperative work and social computing. ACM, 1485-1500.

[5] Robert Asen. 2000. Seeking the "counter" in counterpublics. Communication theory 10, 4 (2000), 424-446.

[6] Karen Barad. 1998. Getting real: Technoscientific practices and the materialization of reality. Differences: a journal of feminist cultural studies 10, 2 (1998), 87-91.

[7] Susan Bordo. 1999. Twilight zones: The hidden life of cultural images from Plato to OJ. Univ of California Press.

[8] Susan Bordo. 2004. Unbearable weight: Feminism, Western culture, and the body. Univ of California Press.

[9] Eileen Boris. 2010. Intimate labors: Cultures, technologies, and the politics of care. Stanford University Press.

[10] danah boyd. 2014. It's complicated: The social lives of networked teens. Yale University Press.

[11] Danah Boyd and Jeffrey Heer. 2006. Profiles as conversation: Networked identity performance on Friendster. In Proceedings of the 39th annual Hawaii international conference on system sciences (HICSS'06), Vol. 3. IEEE, 59c-59c.

[12] Danah Michele Boyd. 2004. Friendster and publicly articulated social networking. In Conference on Human Factors in Computing Systems: CHI'04 extended abstracts on Human factors in computing systems, Vol. 24. 1279-1282.

[13] Wendy Brown. 2003. Neo-liberalism and the end of liberal democracy. Theory \& Event 7, 1 (2003).

[14] Moira Burke and Robert E Kraut. 2014. Growing closer on facebook: changes in tie strength through social network site use. In Proceedings of the SIGCHI conference on human factors in computing systems. ACM, 4187-4196.
[15] Kathy Charmaz. 2006. Constructing grounded theory: A practical guide through qualitative research. (2006).

[16] Hui-Tzu Grace Chou and Nicholas Edge. 2012. "They are happier and having better lives than I am": the impact of using Facebook on perceptions of others' lives. Cyberpsychology, Behavior, and Social Networking 15, 2 (2012), 117-121.

[17] Lisa Cosgrove and Justin M Karter. 2018. The poison in the cure: Neoliberalism and contemporary movements in mental health. Theory \& Psychology 28, 5 (2018), 669-683.

[18] Munmun De Choudhury and Sushovan De. 2014. Mental health discourse on reddit: Self-disclosure, social support, and anonymity. In Eighth International AAAI Conference on Weblogs and Social Media.

[19] Sofia Dewar, Schinria Islam, Elizabeth Resor, and Niloufar Salehi. 2019. Finsta: Creating" Fake" Spaces for Authentic Performance. In Extended Abstracts of the 2019 CHI Conference on Human Factors in Computing Systems. ACM, LBW1214.

[20] Joan Morris DiMicco and David R Millen. 2007. Identity management: multiple presentations of self in facebook. In Proceedings of the 2007 international ACM conference on Supporting group work. ACM, 383-386.

[21] Brooke Erin Duffy and Ngai Keung Chan. 2019. "You never really know who's looking": Imagined surveillance across social media platforms. New Media \& Society 21, 1 (2019), 119-138.

[22] Ana Elias, Rosalind Gill, and Christina Scharff. 2017. Aesthetic labour: Beauty politics in neoliberalism. In Aesthetic labour. Springer, 3-49.

[23] Ana Sofia Elias and Rosalind Gill. 2018. Beauty surveillance: The digital self-monitoring cultures of neoliberalism. European Journal of Cultural Studies 21, 1 (2018), 59-77.

[24] Shelly D Farnham and Elizabeth F Churchill. 2011. Faceted identity, faceted lives: social and technical issues with being yourself online. In Proceedings of the ACM 2011 conference on Computer supported cooperative work. ACM, 359-368.

[25] Nancy Fraser. 1990. Rethinking the public sphere: A contribution to the critique of actually existing democracy. Social text 25/26 (1990), 56-80.

[26] Jeremy Gilbert. 2013. What kind of thing is' neoliberalism'? New Formations 80, 80 (2013), 7-22.

[27] Erving Goffman and others. 1978. The presentation of self in everyday life. Harmondsworth London.

[28] Oliver Haimson. 2018. Social media as social transition machinery. Proceedings of the ACM on Human-Computer Interaction 2, CSCW (2018), 63. 
[29] Oliver L Haimson, Jed R Brubaker, Lynn Dombrowski, and Gillian R Hayes. 2016. Digital footprints and changing networks during online identity transitions. In Proceedings of the $2016 \mathrm{CHI}$ Conference on Human Factors in Computing Systems. ACM, 2895-2907.

[30] Oliver L Haimson and Anna Lauren Hoffmann. 2016. Constructing and enforcing" authentic" identity online: Facebook, real names, and non-normative identities. First Monday 21, 6 (2016).

[31] Arlie Russell Hochschild. 1979. Emotion work, feeling rules, and social structure. American journal of sociology 85, 3 (1979), 551-575.

[32] Bernie Hogan. 2010. The presentation of self in the age of social media: Distinguishing performances and exhibitions online. Bulletin of Science, Technology \& Society 30, 6 (2010), 377-386.

[33] Melissa G Hunt, Rachel Marx, Courtney Lipson, and Jordyn Young. 2018. No more FOMO: Limiting social media decreases loneliness and depression. Journal of Social and Clinical Psychology 37, 10 (2018), 751-768.

[34] Lilly Irani. 2018. "Design Thinking": Defending Silicon Valley at the Apex of Global Labor Hierarchies. Catalyst: Feminism, Theory, Technoscience 4, 1 (2018).

[35] Lilly Irani. 2019. Chasing Innovation: Making Entrepreneurial Citizens in Modern India. Vol. 22. Princeton University Press.

[36] Lilly C Irani and M Silberman. 2016. Stories we tell about labor: Turkopticon and the trouble with design. In Proceedings of the 2016 CHI conference on human factors in computing systems. ACM, 4573-4586.

[37] Adam N Joinson. 2001. Self-disclosure in computer-mediated communication: The role of self-awareness and visual anonymity. European journal of social psychology 31, 2 (2001), 177-192.

[38] Akane Kanai. 2019. On not taking the self seriously: Resilience, relatability and humour in young women's Tumblr blogs. European Journal of Cultural Studies 22, 1 (2019), 60-77.

[39] Hanna Krasnova, Helena Wenninger, Thomas Widjaja, and Peter Buxmann. 2013. Envy on Facebook: a hidden threat to users' life satisfaction? (2013).

[40] Jane Mansbridge. 1996. Using power/fighting power: The polity. Democracy and difference: Contesting the boundaries of the political 46 (1996), 66.

[41] Annette Markham. 2012. Interaction in Digital Contexts: Persistent Characteristics. (24 8 2012). Retrieved September 18, 2019 from

https://annettemarkham. com/2012/08/dramaturgy1/.

[42] Alice E Marwick. 2005. 'I'm a Lot More Interesting than a Friendster Profile': Identity Presentation, Authenticity and Power in Social Networking Services. Association of Internet Researchers 6 (2005).
[43] Alice E Marwick and danah boyd. 2011. I tweet honestly, I tweet passionately: Twitter users, context collapse, and the imagined audience. New media \& society 13, 1 (2011), 114-133.

[44] Kyle Aaron McGregor and Joanna Li. 2019. 73. Fake Instagrams For Real Conversation: A Thematic Analysis of The Hidden Social Media Life of Teenagers. Journal of Adolescent Health 64, 2 (2019), S39-S40.

[45] Angela McRobbie. 2015. Notes on the perfect: Competitive femininity in neoliberal times. Australian feminist studies 30, 83 (2015), 3-20.

[46] Sharan B Merriam and others. 2002. Introduction to qualitative research. Qualitative research in practice: Examples for discussion and analysis 1, 1 (2002), 1-17.

[47] Katherine Mieszkowski. 2003. Faking Out Friendster. Salon. com 14 (2003).

[48] George Monbiot. 2016. Neoliberalism-the ideology at the root of all our problems. The Guardian 15, 04 (2016).

[49] Paul Monckton. 2016. Instagram App Now Allows Multiple Accounts. Here's How To Do It. (2 2016). https://www . forbes. com/sites/paulmonckton/2016/02/09/ how-to-use-multiple-instagram-accounts/\#71cdd5807080

[50] Chip Morningstar and F Randall Farmer. 2008. The lessons of Lucasfilm's Habitat. Journal For Virtual Worlds Research 1, 1 (2008).

[51] Sarah Perez. 2016. Instagram Confirms Test Of Multiple Account Switching On iOS. (2016). https://techcrunch. com/2016/02/05/ instagram-confirms-test-of-multiple-account-switching $\backslash$ -on-ios/

[52] Mikaela Pitcan, Alice E Marwick, and danah boyd. 2018. Performing a vanilla self: Respectability politics, social class, and the digital world. Journal of Computer-Mediated Communication 23, 3 (2018), 163-179.

[53] Bernard Rimé. 2009. Emotion elicits the social sharing of emotion: Theory and empirical review. Emotion review 1, 1 (2009), 60-85.

[54] Oliver Robinson. 2014. Sampling in interview-based qualitative research: A theoretical and practical guide. Qualitative research in psychology 11 (2014).

[55] Ronen Shamir. 2008. The age of responsibilization: On market-embedded morality. Economy and society 37, 1 (2008), 1-19.

[56] Jennifer Sonne and Ingrid Erickson. 2018. The Expression of Emotions on Instagram. In Proceedings of the 9th International Conference on Social Media and Society. ACM, 380-384.

[57] Lucy Suchman. 1993. Working relations of technology production and use. Computer supported cooperative work 2, 1-2 (1993), 21-39. 
[58] Lucy Suchman. 2007. Human-machine reconfigurations: Plans and situated actions. Cambridge University Press.

[59] Lucy Suchman. 2009. Agencies in technology design: Feminist reconfigurations. In Proceedings of 5 th European Symposium on Gender \& ICT, Digital Cultures: Participation-Empowerment-Diversity.

[60] Lucy Suchman and Brigitte Jordan. 1997. Computerization and women's knowledge. Reinventing technology, rediscovering community: Critical explorations of computing as a social practice (1997), 97-105.

[61] Anna Lowenhaupt Tsing. 2015. The mushroom at the end of the world: On the possibility of life in capitalist ruins. Princeton University Press.

[62] Nancy A Van House. 2011. Feminist HCI meets Facebook: Performativity and social networking sites. Interacting with computers 23, 5 (2011), 422-429.

[63] Ron Wakkary and Leah Maestri. 2007. The resourcefulness of everyday design. In Proceedings of the 6th ACM SIGCHI conference on Creativity \& cognition. ACM, 163-172.

[64] Sophie F Waterloo, Susanne E Baumgartner, Jochen Peter, and Patti M Valkenburg. 2018. Norms of online expressions of emotion: Comparing Facebook, Twitter, Instagram, and WhatsApp. new media \& society 20, 5 (2018), 1813-1831.

[65] Emily Weinstein. 2018. The social media see-saw: Positive and negative influences on adolescents' affective well-being. New Media \& Society 20, 10 (2018), 3597-3623.

[66] Jessica Winter. 2013. Selfie-loathing. Slate 23 (2013).

[67] Xuan Zhao, Niloufar Salehi, Sasha Naranjit, Sara Alwaalan, Stephen Voida, and Dan Cosley. 2013. The many faces of Facebook: Experiencing social media as performance, exhibition, and personal archive. In Proceedings of the SIGCHI conference on human factors in computing systems. ACM, 1-10. 